\title{
Global properties of spherical nuclei obtained from Hartree-Fock-Bogoliubov calculations with the Gogny force
}

\author{
M. Kleban and B. Nerlo-Pomorska \\ Katedra Fizyki Teoretycznej, Uniwersytet Marii Curie Skłodowskiej, PL-20031 Lublin, Poland \\ J. F. Berger, J. Dechargé, M. Girod, and S. Hilaire \\ Centre d'Etudes de Bruyères-le-Châtel, F-91680 Bruyères-le-Châtel, France
}

(Received 26 September 2001; published 9 January 2002)

\begin{abstract}
Self-consistent Hartree-Fock-Bogoliubov (HFB) calculations have been performed with the Gogny force for nuclei along several constant $Z$ and constant $N$ chains, with the purpose of extracting the macroscopic part of the binding energy using the Strutinsky prescription. The macroscopic energy obtained in this way is compared to current liquid-drop formulas. The evolution of the single-particle levels derived from the HFB calculations along the constant $Z$ and constant $N$ chains and the variations of the different kinds of nuclear radii are also analyzed. Those radii are shown to follow isospin-dependent three parameter laws close to the phenomenological formulas which reproduce experimental data.
\end{abstract}

DOI: 10.1103/PhysRevC.65.024309

PACS number(s): 21.60.Jz, 21.10.Dr, 21.10.Pc

\section{INTRODUCTION}

Microscopic calculations using the effective nucleonnucleon interaction proposed by Gogny have been shown to reproduce in a very satisfactory way a variety of nuclear properties over a wide range of proton and neutron numbers [1]. For instance, binding energies, separation energies of one or two nucleons, and charge and neutron mean square radii usually agree quite well with known experimental data. In view of this, it seems appropriate to use theoretical results obtained in such a framework to derive systematics of nuclear properties that could be useful for making predictions concerning nuclear species or aspects of nuclear structure not yet known, such as neutron distribution radii or binding energies of superheavy nuclei.

In the present work, simple formulas are used in order to represent the binding energies and the charge, proton, and neutron root-mean-square (rms) radii obtained in HartreeFock-Bogoliubov (HFB) calculations with the Gogny force, and they are compared with existing phenomenological formulas. Our aim is to determine simple but realistic enough parametrizations that would avoid performing timeconsuming microscopic calculations when only rough estimates of the binding energies or radii are needed. Some preliminary results have already been published in Refs. [3,4], for a smaller number of nuclei however.

Section II presents a short overview of the theoretical models used in this work. Section III explains how the basis parameters employed in the self-consistent calculations have been determined. In Sec. IV, the results concerning the single-particle level schemes of representative isotonic and isotopic chains are analyzed. Section V gives the method used to subtract the neutron and proton shell and pairing corrections from the HFB energy. The "macroscopic Gogny energy" obtained in this way is then parametrized by means of a liquid-drop-like formula [5]. In Sec. VI, systematics of the mass, charge, proton, and neutron radii and of the ratios of proton to neutron radii are presented. Simple isospin- and mass number-dependent parametrizations of these quantities are determined, which appear in excellent agreement with those derived using the relativistic mean-field method [6]. The formula obtained for the deformation-independent ratios of proton to neutron rms radii is especially useful, since it should be valid for all even-even nuclei, either spherical or deformed. Conclusions and perspectives for further work are gathered at the end of the paper.

\section{THEORY}

The Gogny density-dependent effective nucleon-nucleon force is of the following form [1]:

$$
\begin{aligned}
V_{12}= & \sum_{i=1}^{2}\left(W_{i}+B_{i} \hat{P}_{\sigma}-H_{i} \hat{P}_{\tau}-M_{i} \hat{P}_{\sigma} \hat{P}_{\tau}\right) e^{-\left(\vec{r}_{1}-\vec{r}_{2}\right)^{2} / \mu_{i}^{2}} \\
& +i W_{\mathrm{LS}}\left(\overline{\nabla_{1}-\nabla_{2}}\right) \times \delta\left(\vec{r}_{1}-\vec{r}_{2}\right)\left(\overline{\nabla_{1}-\nabla_{2}}\right) \cdot\left(\vec{\sigma}_{1}+\vec{\sigma}_{2}\right) \\
& +t_{0}\left(1+x_{0} \hat{P}_{\sigma}\right) \delta\left(\vec{r}_{1}-\vec{r}_{2}\right)\left[\rho\left(\frac{\vec{r}_{1}+\vec{r}_{2}}{2}\right)\right]^{\gamma}+V_{\mathrm{Coul}},
\end{aligned}
$$

which represents a central finite-range interaction, a zerorange spin-orbit term, and a zero-range density-dependent interaction, respectively, to which one has to add the Coulomb interaction in the case of protons. The central interaction is made up of two distinct Gaussians with ranges $\mu_{1}$ and $\mu_{2}$ whose values are given below. $\hat{P} \sigma$ and $\hat{P} \tau$ denote the spin and isospin exchange operators, respectively, and $\rho$ represents the total density.

We use the Gogny D1S [2] interaction, the parameters of which are given below:

$$
\begin{gathered}
W_{1}=-1720.30 \mathrm{MeV}, \quad W_{2}=103.639 \mathrm{MeV}, \\
B_{1}=1300.00 \mathrm{MeV}, \quad B_{2}=-163.483 \mathrm{MeV}, \\
H_{1}=-1813.53 \mathrm{MeV}, \quad H_{2}=162.812 \mathrm{MeV},
\end{gathered}
$$




$$
\begin{gathered}
M_{1}=1397.60 \mathrm{MeV}, \quad M_{2}=-223.934 \mathrm{MeV}, \\
\mu_{1}=0.7 \mathrm{fm}, \quad \mu_{2}=1.2 \mathrm{fm}, \\
t_{0}=1390.6 \mathrm{MeV} \mathrm{fm}^{3(1+\gamma)}, \quad x_{0}=1, \\
\gamma=1 / 3, \quad W_{\mathrm{LS}}=130 \mathrm{MeV} \mathrm{fm}^{5} .
\end{gathered}
$$

When pairing correlations are neglected, the HFB approach reduces to the Hartree-Fock $(\mathrm{HF})$ method which determines a self-consistent approximation of the nuclear mean field. The corresponding ground state energy $E_{\mathrm{HF}}$ includes a contribution $E_{\text {shell }}$ from shell effects which can be evaluated by applying the Strutinsky smearing procedure [7] to the HF single-particle level distribution. The remaining part of $E_{\mathrm{HF}}$ can be considered as a macroscopic, liquid-drop-like contribution $\widetilde{E}$. Denoting by $e_{\nu}$ the HF proton or neutron singleparticle energies, the Strutinsky shell correction energy $E_{\text {shell }}$ is

$$
E_{\text {shell }}=\sum_{\nu} 2 e_{\nu}-\widetilde{E}
$$

where $\widetilde{E}$ is the smoothed energy

$$
\widetilde{E}=2 \int_{-\infty}^{\lambda} e \bar{\rho}(e) d e .
$$

With the Strutinsky prescription, the smoothed density $\bar{\rho}$ is given by

$$
\bar{\rho}(e)=\frac{1}{\gamma \sqrt{\pi}} \int_{-\infty}^{+\infty} \rho\left(e^{\prime}\right) e^{-\left(e-e^{\prime} / \gamma\right)^{2}} f\left(\frac{e-e^{\prime}}{\gamma}\right) d e^{\prime},
$$

where $\lambda$ is the Fermi energy, $f$ the Strutinsky (sixth order) curvature correction polynomial, and $\gamma$ the width over which smoothing of the single-particle level scheme is performed. The reliability of this procedure requires that $E_{\text {shell }}$ displays a plateau when drawn as a function of $\gamma$, separately for protons and neutrons. A crucial parameter in this respect is the number of single-particle levels taken into account in the evaluation of the shell correction. This problem will be discussed in Sec. V.

Subtracting from the Hartree-Fock energy the shell correction energies of neutrons $E_{\text {shell }}^{n}$ and of protons $E_{\text {shell }}^{p}$, one gets the following estimate of the macroscopic part of the binding energy:

$$
E_{\mathrm{macr}}=E_{\mathrm{HF}}-E_{\mathrm{shell}}^{n}-E_{\mathrm{shell}}^{p} .
$$

Another correction to the macroscopic part of the binding energy is the contribution $E_{\text {pair }}$ of pairing correlations. This correction has been calculated for each nucleus by taking the difference between the energy $E_{\mathrm{HFB}}$ obtained in a full HFB calculation with the Gogny force and the energy $E_{\mathrm{HF}}$ computed using the simple HF method:

$$
E_{\text {pair }}=E_{\mathrm{HFB}}-E_{\mathrm{HF}} \text {. }
$$

The obtained rms radii of the neutron, proton, charge, and mass density distributions are obtained in the microscopic HFB calculations in the usual way. In deformed nuclei, these different radii contain the effect of the quadrupole and other deformations [8]. However, the ratios of proton to neutron radii are almost deformation independent since the proton and neutron density distributions are very similarly deformed.

Starting from the HFB mean square radii $\left\langle r^{2}\right\rangle$, equivalent spherical liquid-drop radii $R$ can be defined through the expression

$$
R=\sqrt{\frac{5}{3}} \sqrt{\left\langle r^{2}\right\rangle}
$$

which follows from the formula giving the mean square radius of a uniform spherical density distribution of radius $R$ :

$$
\left\langle r^{2}\right\rangle=\frac{3}{5} R^{2}
$$

These equivalent radii have then been fitted to a three parameter formula, with explicit isospin and $A$ dependencies,

$$
R=r_{00}\left(1+\alpha \frac{N-Z}{A}+\frac{\beta}{A}\right) A^{1 / 3}=r_{0}(A, I) A^{1 / 3},
$$

similar to the formula used in Ref. [9] in the analysis of the radii obtained from relativistic mean-field calculations. As the ratios of the proton and neutron rms radii are almost deformation independent, they could be well approximated by the following formula depending only on the numbers of protons and neutrons:

$$
\frac{r_{p}}{r_{n}}=c\left(1+a \frac{N-Z}{A}+\frac{b}{A}\right) .
$$

This last relation, together with the effect of the non-pointlike charge distribution of the proton evaluated with the approximate formula

$$
\left\langle r_{\mathrm{ch}}^{2}\right\rangle=\left\langle r_{p}^{2}\right\rangle+0.64 \mathrm{fm}^{2},
$$

can be useful for estimating the neutron radius of a nucleus when its rms charge radius $r_{\mathrm{ch}}=\sqrt{\left\langle r_{\mathrm{ch}}^{2}\right\rangle}$ is known:

$$
r_{n}=\frac{\sqrt{\left\langle r_{\mathrm{ch}}^{2}\right\rangle-0.64 \mathrm{fm}^{2}}}{c\left(1+a \frac{N-Z}{A}+\frac{b}{A}\right)} .
$$

\section{PARAMETERS OF THE CALCULATION}

The nuclei studied in the present work are represented by crosses and dots in the $(N, Z)$ plot of Fig. 1. These are the nuclei close to magic proton and neutron numbers and along the $\beta$-stability line whose ground states are expected to be spherical [10]. They include $\mathrm{Ca}, \mathrm{Sr}, \mathrm{Sn}, \mathrm{Sm}, \mathrm{Pb}$, and $\mathrm{Th}$ isotopes, the $N=50,82$, and 126 isotone chains between the proton and neutron drip lines, and a few $\beta$-stable spherical 


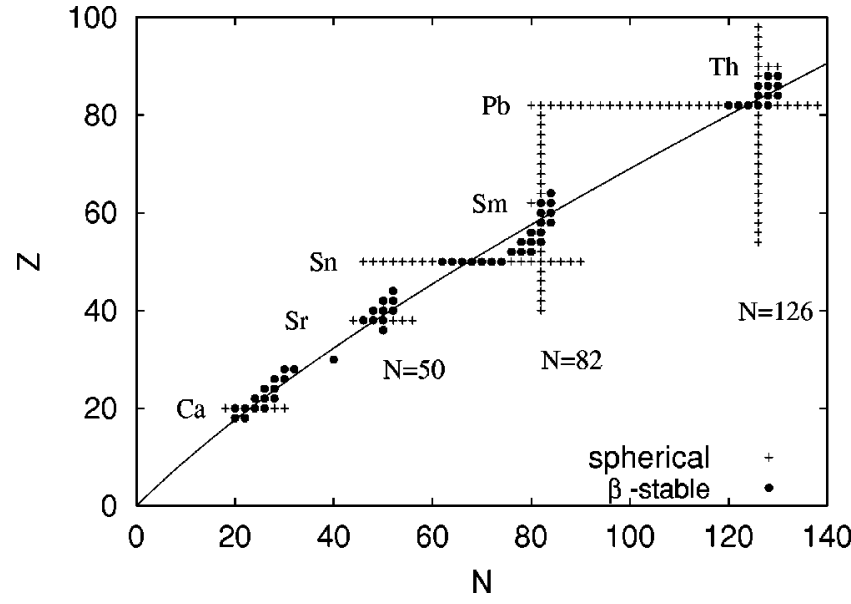

FIG. 1. $(N, Z)$ plot of the spherical nuclei analyzed in the present work. The circles indicate the $\beta$-stable nuclei and the crosses a few representative isotope and isotone chains.

nuclei between $A=38$ and $A=218$.

In the microscopic HF and HFB calculations, the selfconsistent equations have been solved in matrix form by expanding the single-particle or quasiparticle states on finite bases made of spherical harmonic oscillator (HO) eigenfunctions. These bases depend on two parameters: the number $N_{0}$ of major $\mathrm{HO}$ shells included in the bases and the oscillator parameter $\hbar \omega$.

In the present study, bases with $N_{0}=12,14,16$, or 18 major shells have been taken into account, depending on the nucleus under study, the criterion being that $N_{0}$ is large enough to ensure convergence of the HFB energy $\left(E_{\mathrm{HFB}}\right)$ within a few $\mathrm{keV}$. For each nucleus and each value of $N_{0}$, the parameter $\hbar \omega$ has been chosen as the value $\hbar \omega_{\min }$ that minimizes the HF energy $E_{\mathrm{HF}}$. The values of $\hbar \omega_{\min }$ obtained for $\mathrm{Ca}, \mathrm{Sn}$, and $\mathrm{Pb}$ isotopes, and for the $N=126$ isotones are plotted in Fig. 2 as a function of $A$ for $N_{0}=14$, a value of $N_{0}$ large enough for all these nuclei. The error bars indicate the ranges of $\hbar \omega$ for which the variation of $E_{\mathrm{HF}}$ does not exceed $100 \mathrm{keV}$. One can see that, with this number of HO shells, relatively large changes in $\hbar \omega$ do not significantly affect calculated values of $E_{\mathrm{HF}}$. Consequently, approximate analytical interpolation formulas can be used.

A large scale investigation of the values found in spherical nuclei shows that an interpolation formula such as

$$
\hbar \omega_{\min }=n\left(1+k \frac{N-Z}{A}\right) A^{-1 / 3}
$$

can be adopted for all even-even nuclei, with $n$ and $k$ depending on the number $N_{0}$ of shells included in the bases. In the present work, all the obtained values of $\hbar \omega_{\text {min }}$ could be fitted with the values $n=64.05 \mathrm{MeV}$ and $k=-0.46$. An even more accurate interpolation formula, represented by the solid curve in Fig. 2, has been obtained for the specific set of nuclei for which $N_{0}=14$ is found to be a sufficiently large number:

$$
\hbar \omega_{\min }=\left(0.0002 A^{2}-0.1 A+21.1\right) \mathrm{MeV} .
$$

This formula gives a better agreement to calculated $\hbar \omega_{\text {min }}$ for the $\mathrm{Ca}$ up to the $\mathrm{Pb}$ isotopes and for the $N=126$ isotones.

\section{SINGLE-PARTICLE LEVELS}

In order to better visualize the origin of shell effects in the nuclei considered in this work, the Hartree-Fock singleparticle energies obtained with the Gogny force are plotted in Figs. 3-5 for neutrons (left hand sides) and protons (right hand sides). The three figures correspond to $N=50, N=82$, and $N=126$ isotones, respectively, and the levels are drawn as functions of the proton number $Z$. They are labeled with the usual radial quantum numbers $n$, orbital angular momentum $l$, and total angular momentum $j$. The numbers $N_{S}$ be-

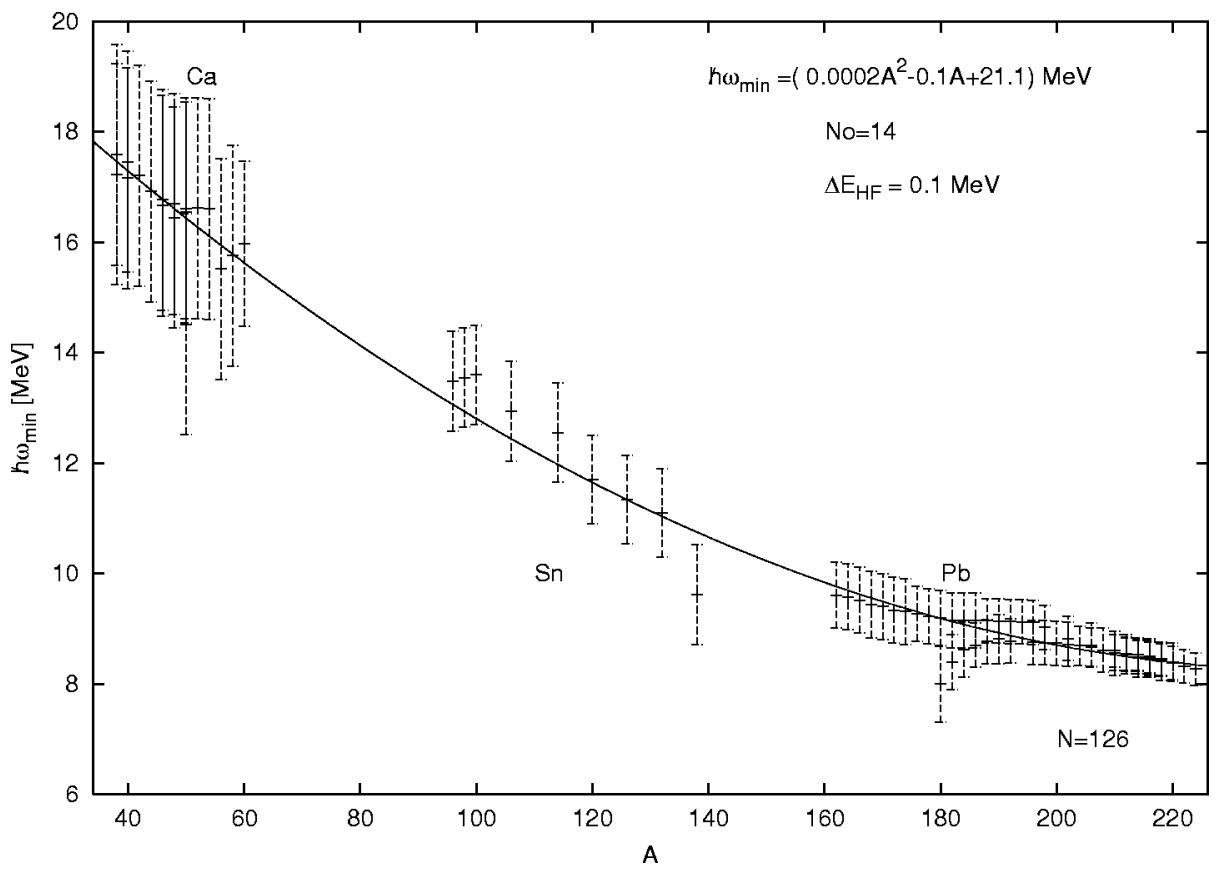

FIG. 2. The dependence on $A$ of the parameter $\hbar \omega_{\min }$ of the $N_{0}$ $=14 \mathrm{HO}$ bases employed in $\mathrm{Ca}$, $\mathrm{Sn}$, and $\mathrm{Pb}$ isotopes. The error bars indicate ranges of $\hbar \omega$ for which the variation of the HF energy does not exceed $0.1 \mathrm{MeV}$. 


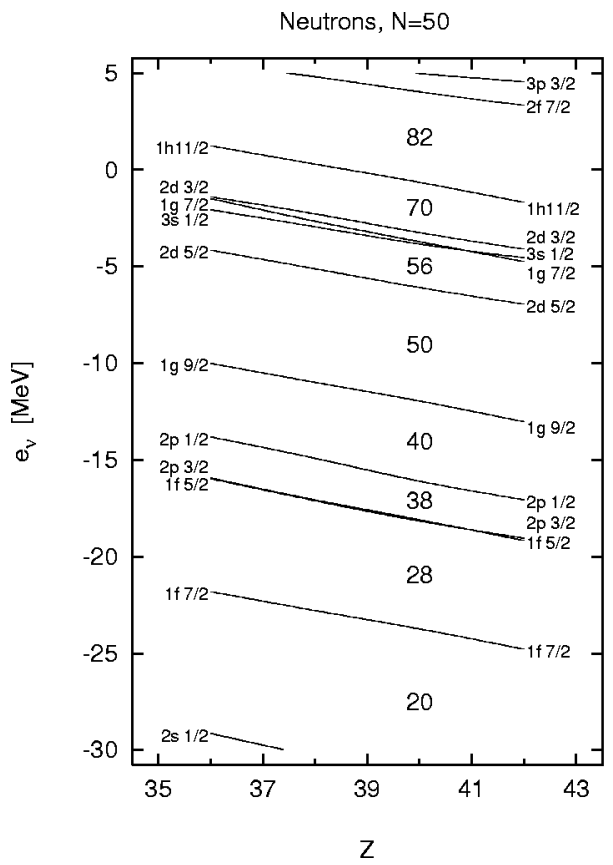

tween the levels indicate the number of nucleons necessary to fill all the levels located below.

One can see that, in addition to the magic shells corresponding to $N_{S}=20,28,50,82,126$, and 184, well-marked subshells appear depending on the number of neutrons $N$. For instance, subshells with $N_{S}=40$ are clearly visible in the neutron and proton level schemes of the $N=50$ and $N=82$ isotones, and $N_{S}=64$ also appears as a subshell in the $N$ $=126$ isotones.

In Figs. 6-11 the neutron (left hand sides) and proton (right hand sides) single-particle levels of the $\mathrm{Ca}, \mathrm{Sr}, \mathrm{Sn}, \mathrm{Sm}$, $\mathrm{Pb}$ and $\mathrm{Th}$ isotopes are plotted as functions of the neutron number $N$. Subshells corresponding to $N_{S}=40,64$, or 100 can also be observed in these figures besides the usual magic
Protons, $\mathrm{N}=50$

FIG. 3. The neutron (left) and proton (right) single-particle levels in $N=50$ isotones versus the proton number $Z$.

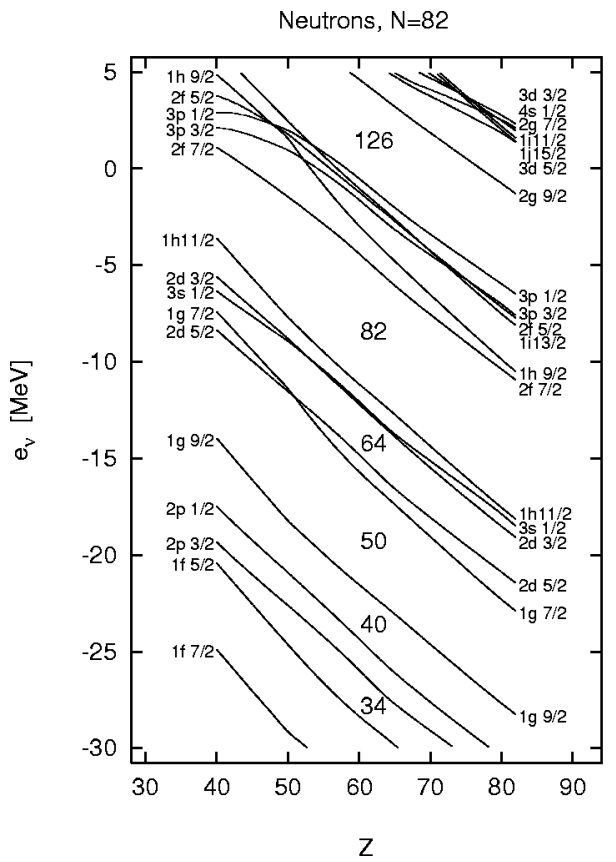

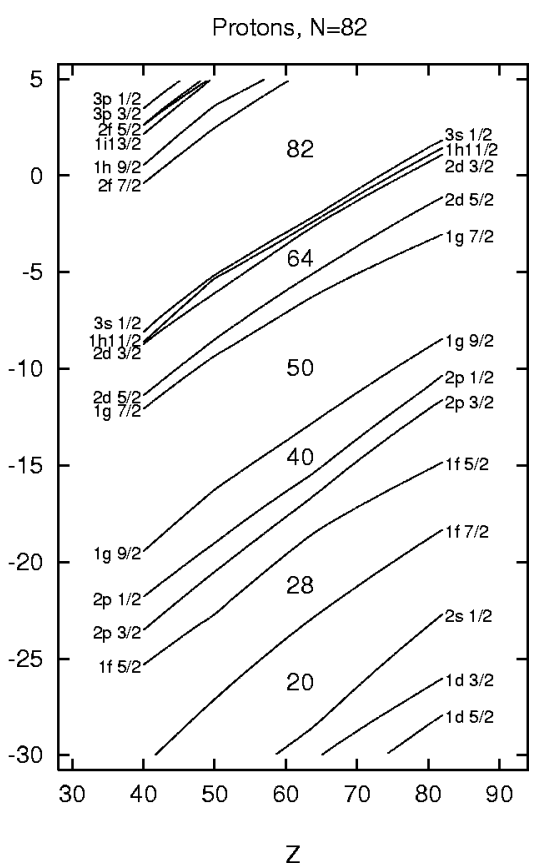

$024309-4$
FIG. 4. The neutron (left) and proton (right) single-particle levels in $N=82$ isotones versus the proton number $Z$. 


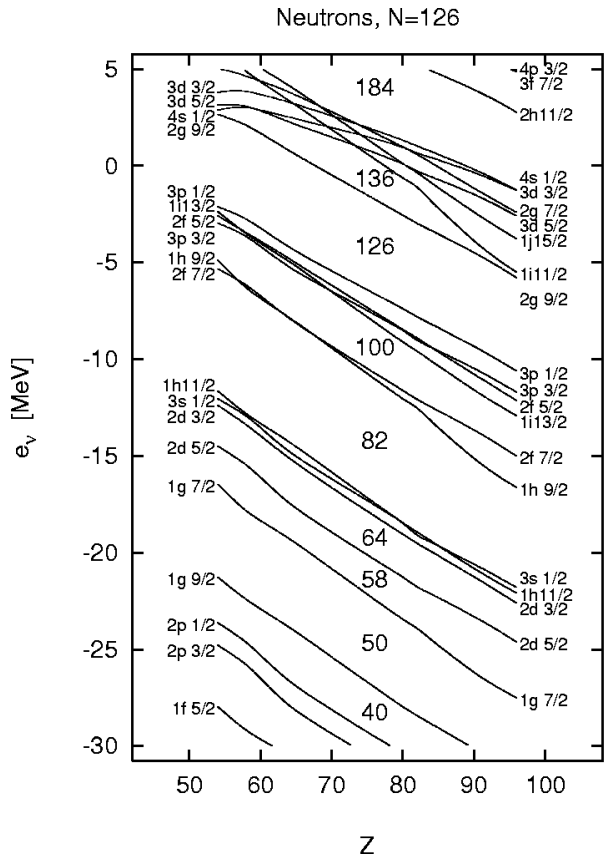

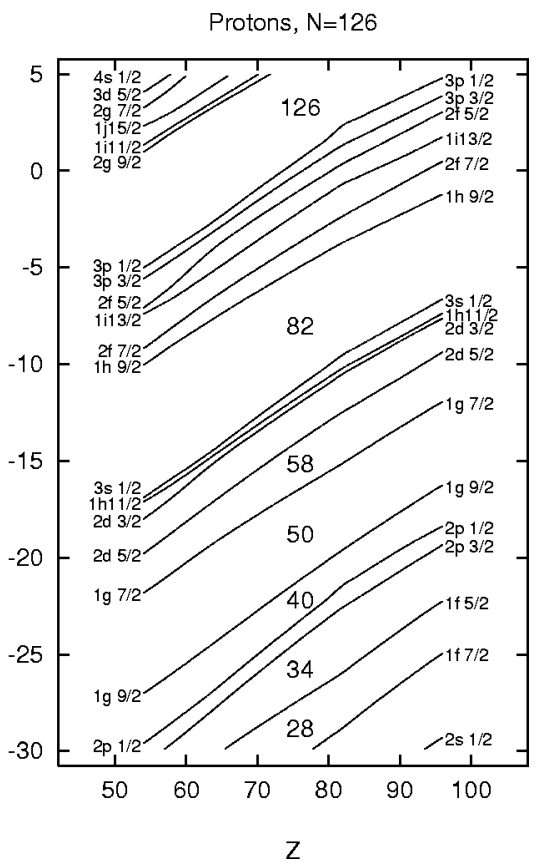

FIG. 5. The neutron (left) and proton (right) single-particle levels in $N=126$ isotones versus the proton number $Z$. of $A$ and a shell correction reflecting the neutron and proton shells. The method of Strutinsky is a subtle technique which demands a very careful choice of the number of singleparticle states above the Fermi level included in the smoothing procedure so as to obtain a plateau in the variations of the shell corrections with respect to the smoothing width $\gamma$. In particular, including too many single-particle states with positive energies strongly affects the determination of the shell effects associated with occupied orbitals. This is so because single particle states with positive energies are obtained in the present microscopic approach as a discrete set whose energy spectrum and shell structure can strongly depend on the choice of basis parameters.
For this reason, the HF single-particle proton and neutron levels included in the Strutinsky smoothing procedure have been chosen in the following way: For lighter nuclei, a cutoff in the single-particle energy has been introduced, having the usual value $\lambda+2 \hbar \omega_{0}$, with $\lambda$ the proton or neutron Fermi energy and $\hbar \omega_{0}$ the average spacing between major shells. The number of single-particle levels taken into account in this way is of the order of 30 , with maximum energies 5 $\mathrm{MeV}$ for neutrons and $15 \mathrm{MeV}$ for protons above the Fermi level. In heavier nuclei, the number of levels included in the smoothing procedure was increased to 50, which corresponds to a cutoff in the single-particle energies of the order of $\lambda+4 \hbar \omega_{0}$, i.e., $15 \mathrm{MeV}$ for neutrons and $30 \mathrm{MeV}$ for

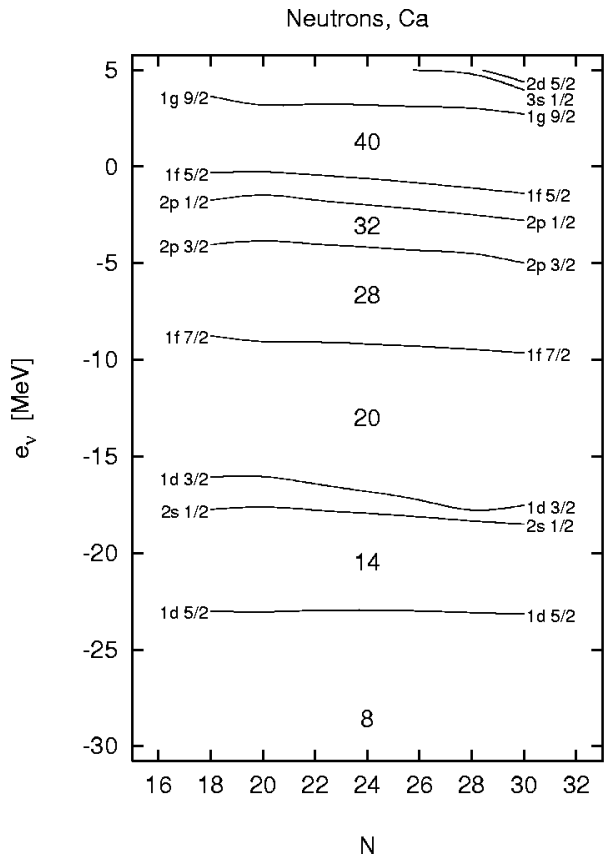

Protons, $\mathrm{Ca}$

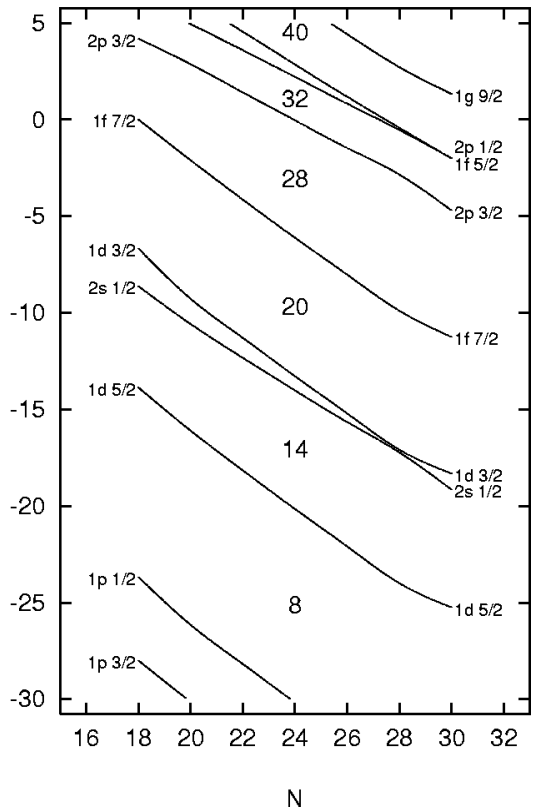

FIG. 6. The neutron (left) and proton (right) single-particle levels in $\mathrm{Ca}$ isotopes versus the neutron number $N$. 


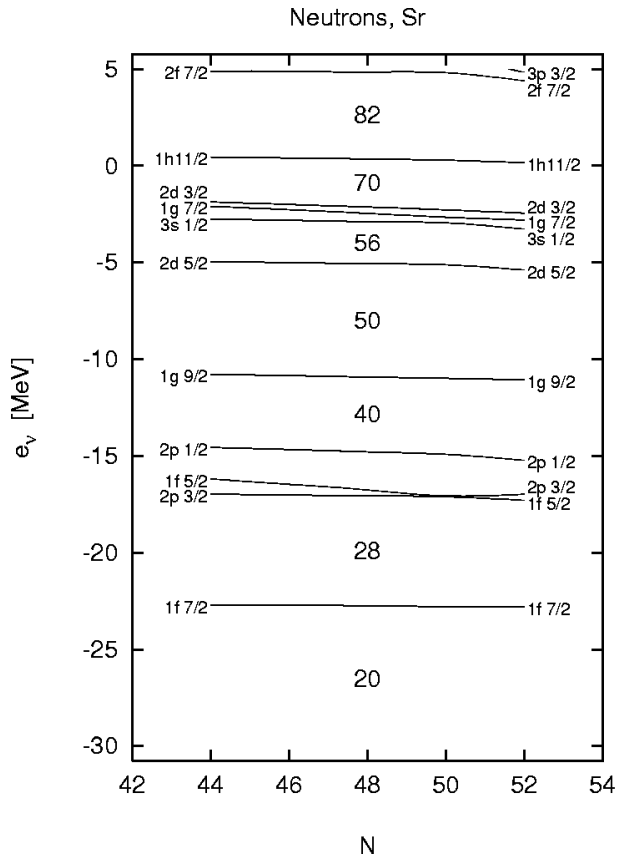

Protons, $\mathrm{Sr}$

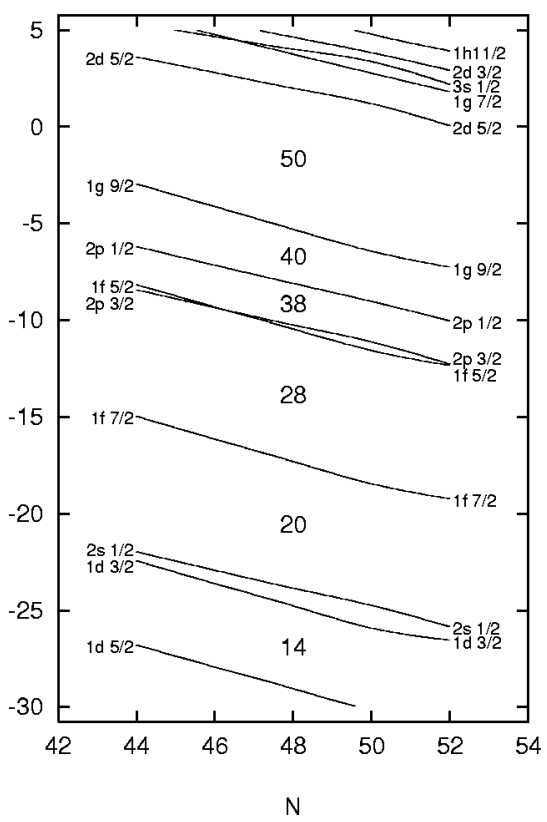

FIG. 7. The neutron (left) and proton (right) single-particle levels in $\mathrm{Sr}$ isotopes versus the neutron number $N$. protons. These numbers have been found to provide a satisfactory stabilization of shell corrections in all the nuclei studied in this work.

In order to find a plateau in the variations of the shell corrections with respect to the width $\gamma$ appearing in Eq. (5), $\gamma$ has been varied, taking as a scale the average shell spacing $\hbar \omega_{0}=40 A^{-1 / 3} \mathrm{MeV}$ [11]. In fact Strutinsky calculations generally obtain a plateau in the shell correction energy of both protons and neutrons for $\gamma$ in the vicinity of the traditional value $\gamma=1.2 \hbar \omega_{0}$ [11]. Figures 12 and 13 give examples of the variations with $\gamma /\left(\hbar \omega_{0}\right)$ of the shell corrections obtained in the present work. Similar behaviors are found in all the nuclei displayed in the $(N, Z)$ plot of Fig. 1. In Fig. 12, the neutron (solid lines) and proton (dashed lines) shell corrections of twelve $N=82$ isotones ranging from $\mathrm{Nd}$ to $\mathrm{Pb}$ are plotted as functions of $\gamma /\left(\hbar \omega_{0}\right)$. In Fig. 13, the different curves represent the shell corrections for neutrons (top) and protons (bottom) calculated in $N=82$ (left) and $N$ $=126$ (right) isotones. One can see that, except for a few cases, as in the proton shell corrections in ${ }^{196} \mathrm{Yb}$ and ${ }^{198} \mathrm{Dy}$, reasonable plateaus are always found around the value $\gamma$ $=1.2 \hbar \omega_{0}$. The results shown in the next figures are those obtained with the latter value of the averaging width $\gamma$.

In the upper part of Fig. 14 the neutron and proton (dashed lines), and total (solid lines) shell corrections are drawn as functions of $A$ for the $\mathrm{Ca}$ up to Th isotopes (left), $N=50,82$ and 126 isotones (center) and $\beta$-stable nuclei (right) included in this study. The lower part of this figure

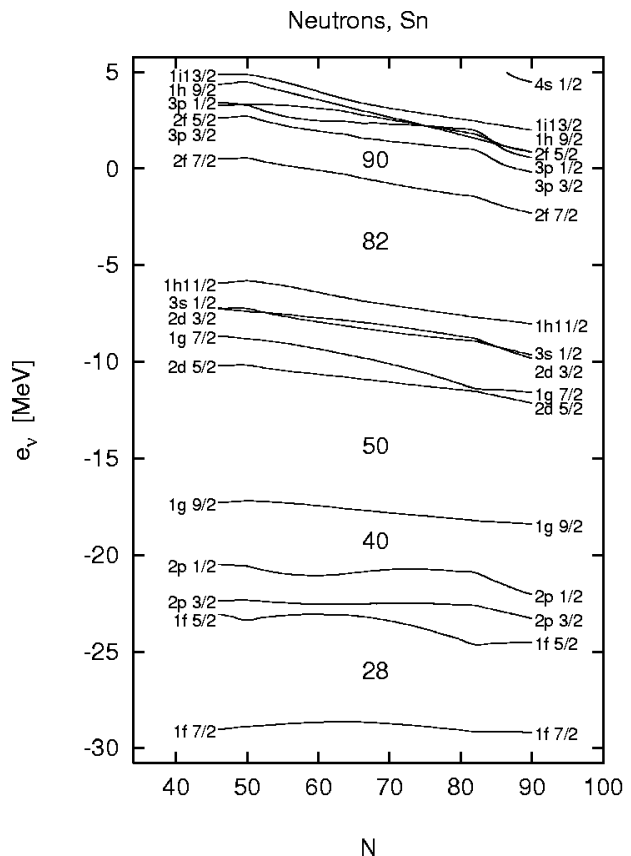

N

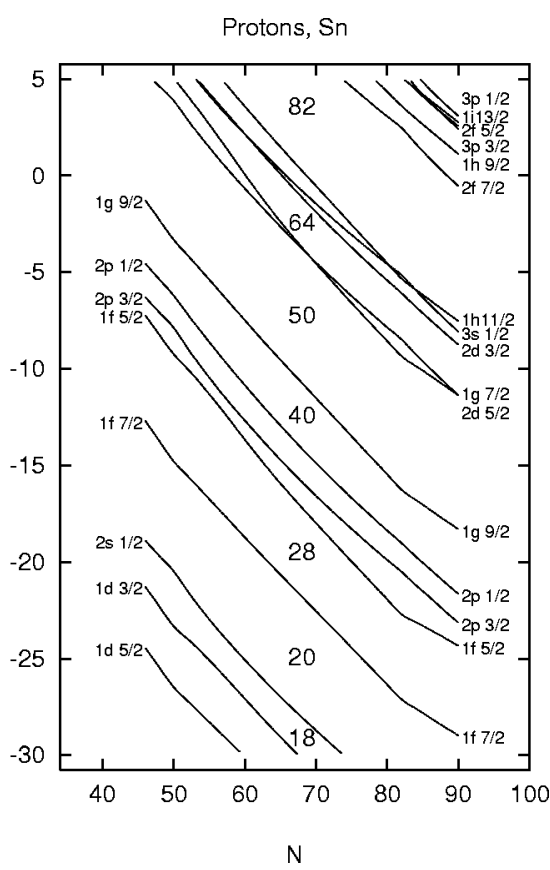

$\mathrm{N}$
FIG. 8. The neutron (left) and proton (right) single-particle levels in Sn isotopes versus the neutron number $N$. 


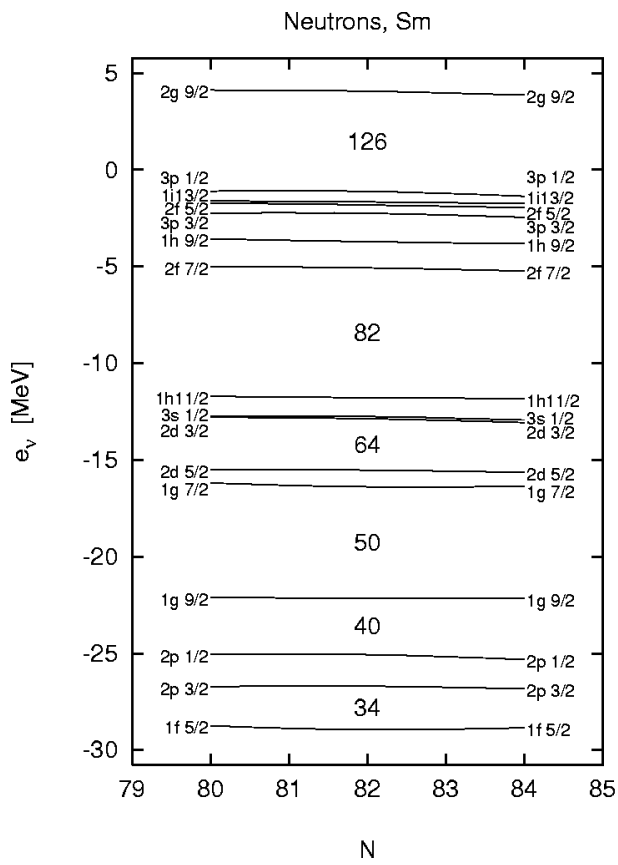

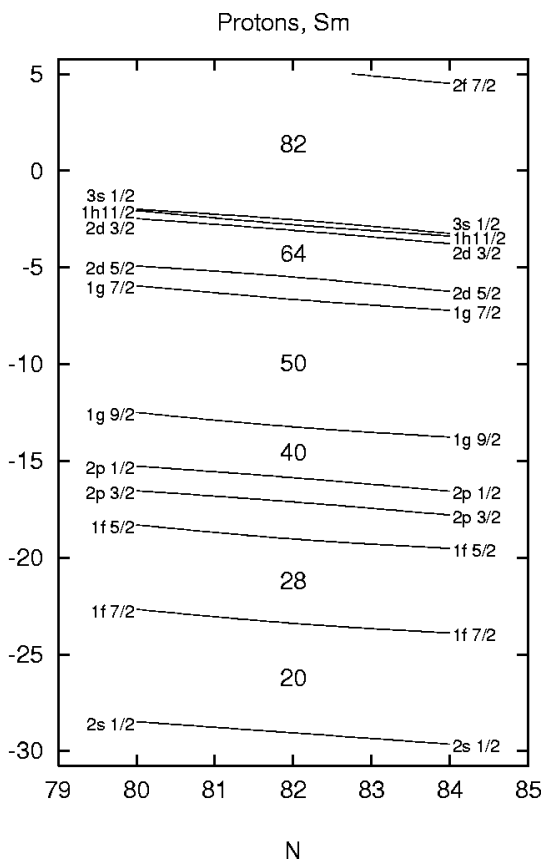

FIG. 9. The neutron (left) and proton (right) single-particle levels in Sm isotopes versus the neutron number $N$. displays the corresponding pairing corrections calculated from Eq. (7) . One observes that the variations of the shell corrections nicely reproduce the expected pattern, with minima located at magic proton and neutron numbers. Neutron shell corrections are seen to be almost independent of the nucleus neutron number $N$, as they should, and proton shell corrections are found practically independent of $Z$.

The macroscopic energies (6) obtained by subtracting the proton and neutron shell corrections from the Hartree-Fock energies $E_{\mathrm{HF}}$ for all the nuclei displayed in Fig. 1 are represented by lines in the three upper plots of Fig. 15. A fit of these macroscopic energies using a liquid-drop formula of the form proposed by Myers and Swiątecki [5] yields the following expression:

$$
\begin{aligned}
E_{\mathrm{LD}}= & {\left[15.65\left(1-1.92 I^{2}\right) A-18.92\left(1-2.1 I^{2}\right) A^{2 / 3}\right.} \\
& \left.-0.73 \frac{Z^{2}}{A^{1 / 3}}+1.99 \frac{Z^{2}}{A}\right] \mathrm{MeV},
\end{aligned}
$$

with $I=(N-Z) / A$. The numerical parameters in this formula are in good agreement with those of Myers and Swiastecki (MS) [5], except for the coefficient in the last term: 1.99 instead of 1.21. This term represents the correction to the Coulomb energy of a charge liquid drop that accounts for the diffuseness of the proton density distribution. The correction appears to be larger in the case of formula (16) - the differ-

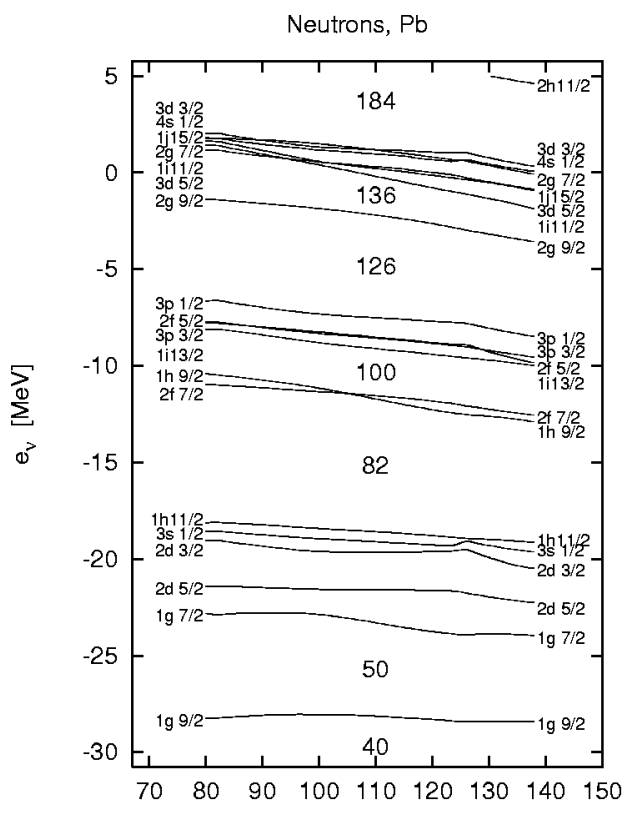

N
Protons, $\mathrm{Pb}$

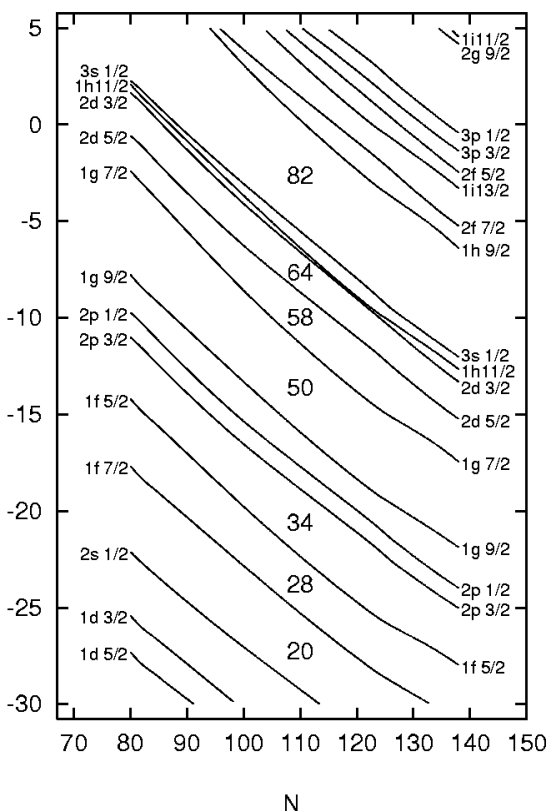

N
FIG. 10. The neutron (left) and proton (right) single-particle levels in $\mathrm{Pb}$ isotopes versus the neutron number $N$. 


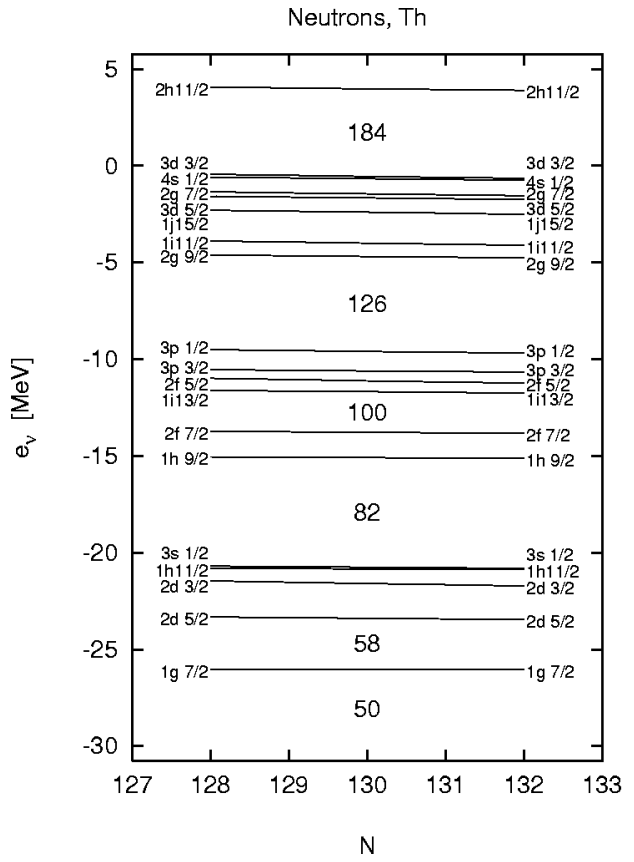

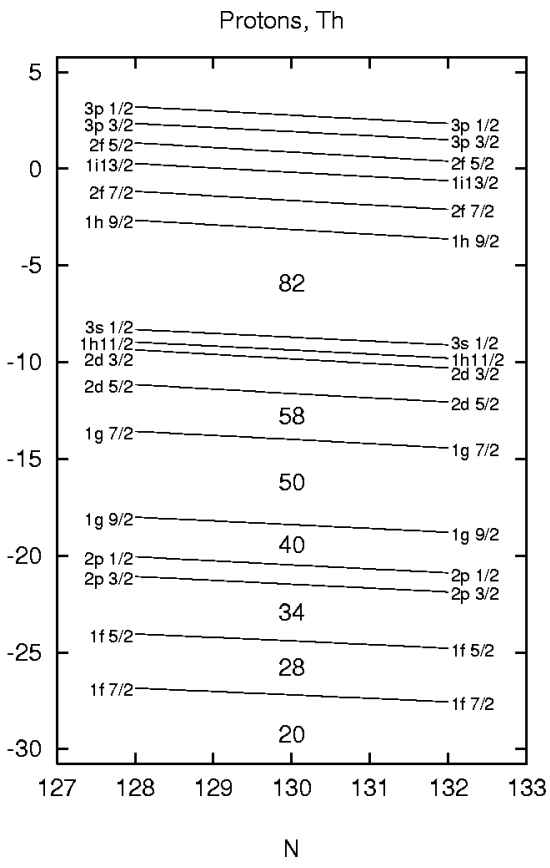

FIG. 11. The neutron (left) and proton (right) single-particle levels in Th isotopes versus the neutron number $N$. ence with the equivalent $\mathrm{MS}$ term reaches $\simeq 30 \mathrm{MeV}$ in ${ }^{252} \mathrm{Fm}$ - which compensates for the fact that the Coulomb energy derived from the self-consistent results is larger than the MS one. Let us also note that the asymmetry coefficients in the volume and surface terms of Eq. (16) - the coefficients of $I^{2}$ - are not equal, contrary to what is assumed in the MS formula. On the other hand, the parameters appearing in Eq. (16) are closer to those of the most recent best empirical fits, where the whole set of presently known nuclear masses and fission barriers is used, than to those of the various formulas derived from liquid-drop models [12].

The fitted macroscopic energy (16) and the calculated values $E_{\text {macr }}$ are completely superimposed in the three upper plots of Fig. 15. In order to better appreciate the quality of the fit given by Eq. (16), the differences $E_{\text {macr }}-E_{\mathrm{LD}}$ are displayed in the lower part of the figure, using an enlarged energy scale. One observes that these differences do not exceed $3 \mathrm{MeV}$, which represents less than $0.5 \%$ of the total $E_{\mathrm{HF}}$ energy.

\section{RADII}

In order to derive systematics for the radii of all the nuclei displayed in Fig. 1, Eq. (8) has been used to extract from the different-neutron, proton, mass, and charge-Hartree-FockBogoliubov rms radii, corresponding to isospin-dependent radius constants $r_{0}^{n}, r_{0}^{p}, r_{0}^{\text {tot }}$, and $r_{0}^{\text {ch }}$. Namely, each kind of microscopic rms radius is multiplied by $\sqrt{5 / 3} A^{-1 / 3}$. A fit of the obtained values with formula (10) yielded the following parametrizations:

$$
r_{0}^{n}=1.17(1+0.12 I+3.29 / A) \quad \mathrm{fm},
$$
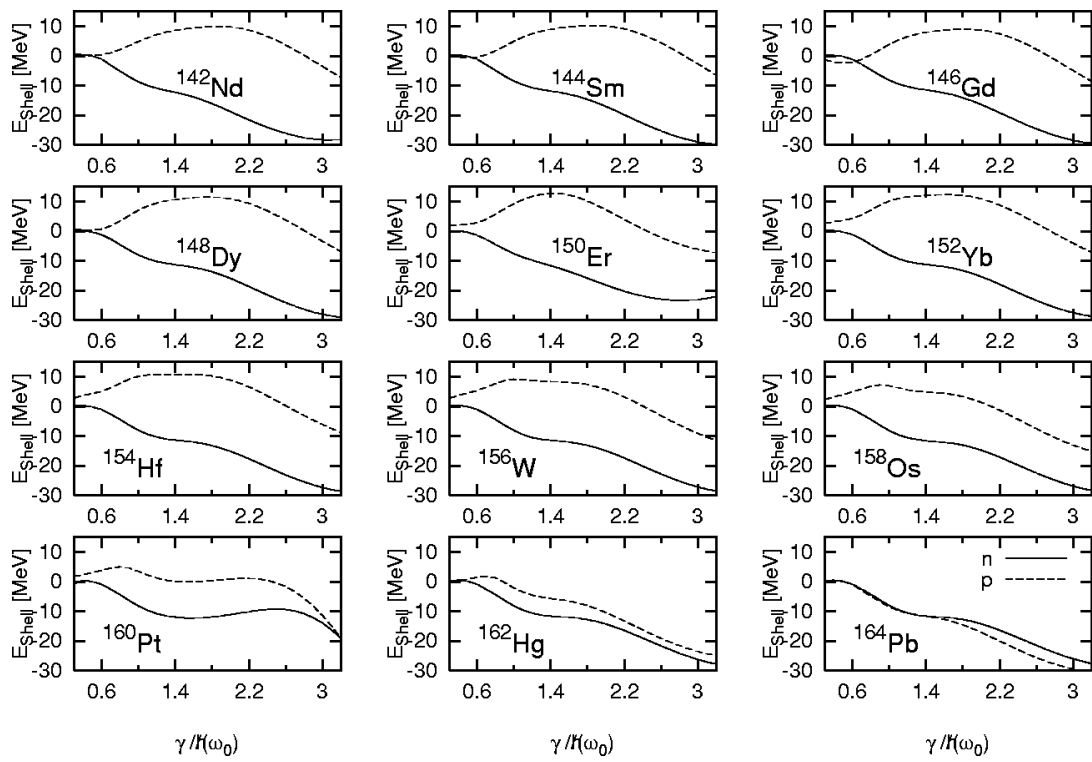
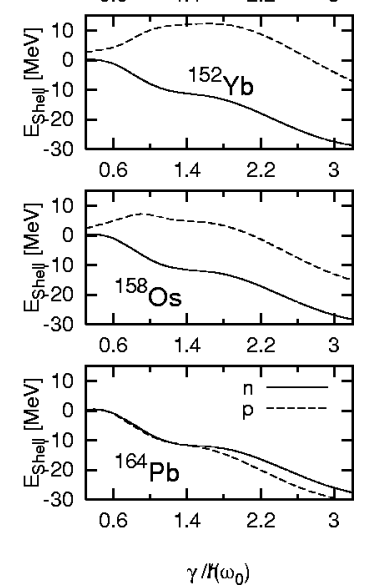

FIG. 12. Proton (dashed lines) and neutron (solid lines) shell corrections of the $\mathrm{Nd}$ up to $\mathrm{Pd}$ $N=82$ isotones as functions of $\gamma /\left(\hbar \omega_{0}\right)$ with $\hbar \omega_{0}=40 A^{-1 / 3} \mathrm{MeV}$. 

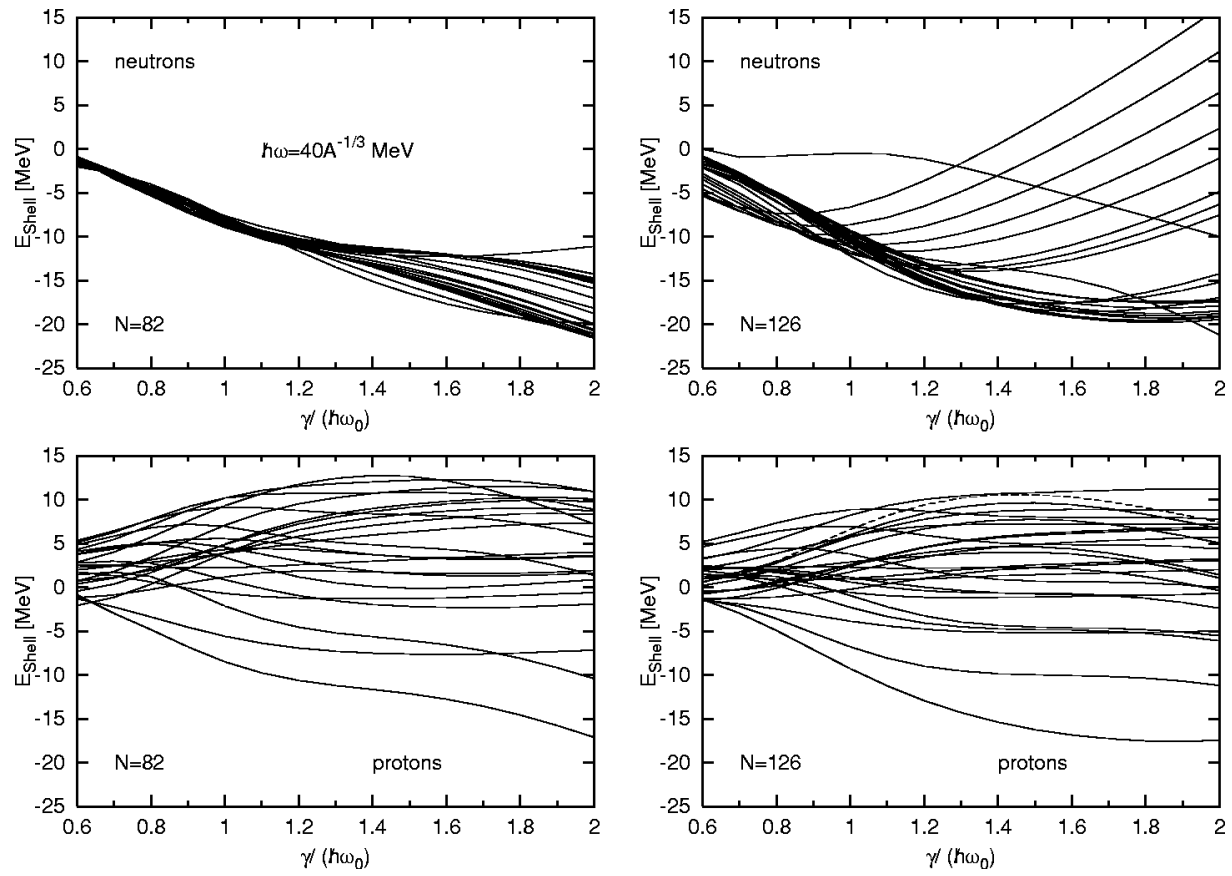

FIG. 13. Shell corrections for neutrons (upper row) and protons (lower row) of $N=82$ (left) and $N=126$ (right) isotones versus $\gamma /\left(\hbar \omega_{0}\right) \quad$ with $\quad \hbar \omega_{0}$ $=40 A^{-1 / 3} \mathrm{MeV}$.

$$
\begin{aligned}
& r_{0}^{p}=1.21(1-0.14 I+1.83 / A) \quad f m, \\
& r_{0}^{\mathrm{tot}}=1.19(1+0.03 I+2.70 / A) \quad \mathrm{fm}, \\
& r_{0}^{\mathrm{ch}}=1.22(1-0.15 I+2.32 / A) \quad \mathrm{fm} .
\end{aligned}
$$

It is interesting to observe that the leading coefficient in Eq. (19) is almost equal to the usual value $r_{0}=1.2 \mathrm{fm}$, although the $I$ and $A$ dependencies included in the next terms may lead to significant deviations in the case of light or exotic nuclei.

The above parametrizations appear very close to those derived in Ref. [3] by taking into account the $\mathrm{Ca}, \mathrm{Sr}, \mathrm{Sn}, \mathrm{Sm}$, and $\mathrm{Pb}$ isotopes only, and to those obtained in the analysis of the rms radii calculated in the framework of the relativistic mean-field theory for a set of nuclei similar to the one envisaged here [9].

Using formula (11), the ratios of proton to neutron radii could be fitted with the parametrization

$$
\frac{r_{p}}{r_{n}}=1.04(1-0.27 I-1.12 / A) .
$$

This parametrization again appears very close to those derived in the studies of Refs. $[3,4,9]$ mentioned above.

Let us point out that the parametrization given by formula (21), which has been established for the spherical nuclei shown in Fig. 1, has been found later on to reproduce the ratios of the HFB proton to neutron radii of all nuclei between the proton and neutron drip lines, spherical or deformed, in the range $20<A<318$. This latter result follows from large scale HFB calculations with axial symmetry presently under way with the Gogny force.

The results obtained in the present work are summarized in Figs. 16 and 17. The upper part of Fig. 16 displays the mass HFB radii (solid lines) at constant $Z$ (left), constant $N$ (center), and for $\beta$-stable (right) nuclei, together with the fits
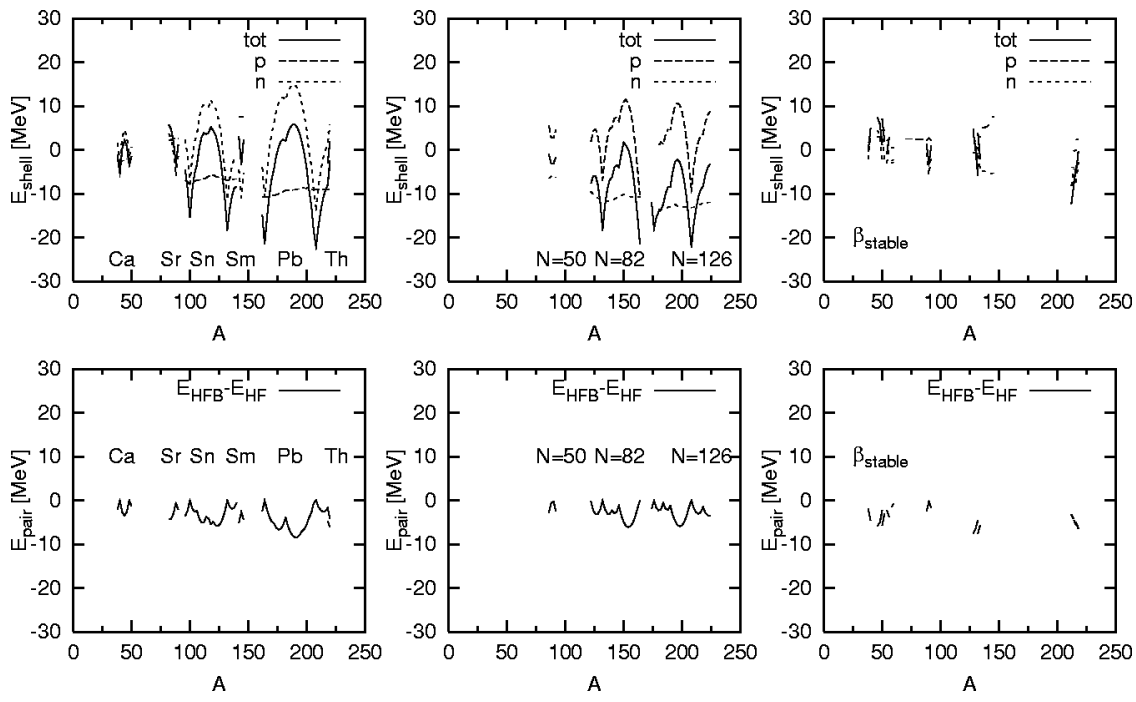

FIG. 14. The upper row shows the total (solid lines), and neutron and proton (dashed lines) shell corrections of $\mathrm{Ca}$ up to $\mathrm{Th}$ isotopes (left), $N=50, N=82$, and $N=126$ isotones (center), and $\beta$-stable nuclei (right) versus the mass number $A$. The lower row displays the pairing corrections calculated as the differences $E_{\text {pair }}$ $=E_{\mathrm{HFB}}-E_{\mathrm{HF}}$ for the same nuclei. 

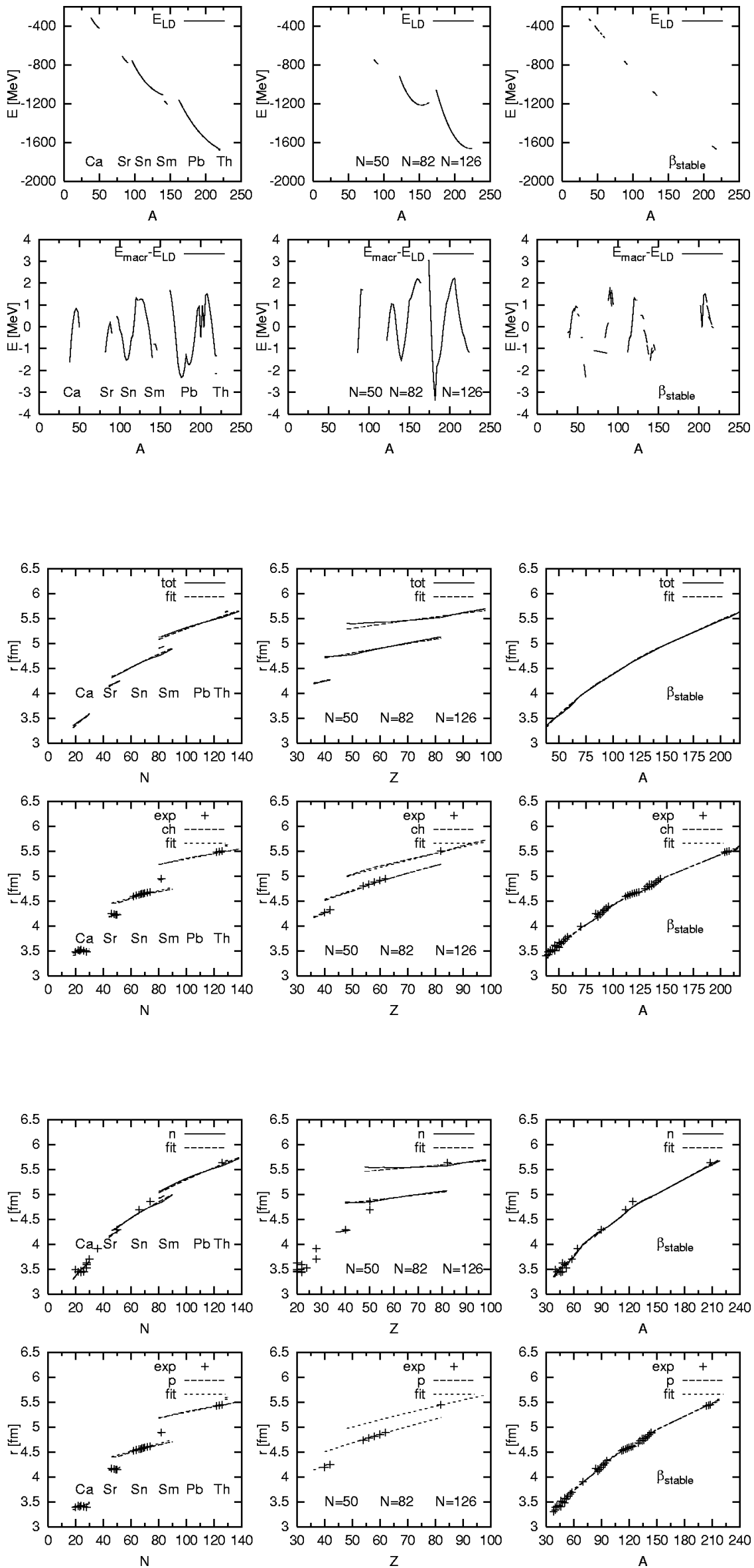

FIG. 15. The fitted liquid-drop energy $E_{\mathrm{LD}}$ given by Eq. (16) (solid lines) for constant $Z$ (left), constant $N$ (center), and $\beta$-stable (right) nuclei versus their mass number $A$. The differences $E_{\text {macr }}$ $-E_{\mathrm{LD}}$ are shown in the three lower plots for the same nuclei.
FIG. 16. Upper row: total HFB rms radii (solid lines), and their fits with formula (19) (dashed lines) in constant $Z$ (left), constant $N$ (center), and $\beta$-stable (right) nuclei. Lower row: HFB charge radii (dashed lines) compared with experimental data (Ref. [13]) (crosses) and with the fit given by formula (20).
FIG. 17. Neutron (upper row, solid lines) and proton (lower row, dashed lines) HFB rms radii of constant $Z$ (left), constant $N$ (center), and $\beta$-stable (left) nuclei compared with experimental data (Refs. [13,14]) (crosses) and with formulas (17) and (18) (dashed lines). 
given by Eq. (19). The lower part of the figure shows in a similar way the results obtained for charged radii. The fits are given by Eq. (20), and experimental data, taken from Ref. [13], have been indicated by crosses. Calculated charge radii appear in good agreement with experimental ones, except in a few cases. One must stress in this respect that the charge radii obtained in the HFB approach do not include the influence of the long range correlations associated with collective oscillations of the mean field, such as random-phase approximation ground state correlations or shape coexistence effects occurring in soft nuclei. As is well known, these correlations beyond the mean field may lead to a significant increase of HFB rms radii.

Figure 17 is the equivalent of Fig. 16 for neutron (upper part) and proton (lower part) radii. Experimental data on proton radii (crosses) have been taken from Refs. [13,14] and the fits are given by formulas (17) and (18).

Finally, as already pointed out in Sec. II, the ratios $r_{p} / r_{n}$ are deformation-independent quantities that can be used to calculate the neutron radii of nuclei whose charged radii are known [see Eq. (13)]. From formula (21), one gets the following expression for neutron radii as functions of $A, I$ $=(N-Z) / A$ and $\left\langle r_{\mathrm{ch}}^{2}\right\rangle$, the mean square charge radius:

$$
r_{n}=\frac{\sqrt{\left\langle r_{\mathrm{ch}}^{2}\right\rangle-0.64}}{1.04(1-0.27 I-1.12 / A)} \mathrm{fm} .
$$

\section{CONCLUSIONS}

In this work, the results of self-consistent HFB calculations performed with the Gogny effective interaction for several isotopic and isotonic chains of spherical nuclei have been analyzed and compared with those given by phenomenological expressions and by the relativistic mean field approach. The more important conclusions that can be drawn from this analysis are the following:

(1) The average binding energy of nuclei calculated by applying the Strutinsky smoothing procedure to the singleparticle level scheme obtained from the HFB self-consistent mean field, Eq. (16), is in excellent agreement with the liquid-drop formula of Myers and Swiątecki (MS) [5]. The main difference occurs in the term correcting the charged liquid-drop Coulomb energy for the diffuseness of the proton density. Also, the asymmetry coefficients in the volume and surface terms are found to be unequal, contrary to the MS formula, and the reduced radius parameter $r_{0}$ is slightly smaller $\left(r_{0}=1.19 \mathrm{fm}\right.$ instead of $\left.r_{0}=1.205 \mathrm{fm}\right)$. When all contributions are added, the differences between Eq. (16) and the MS formula compensate, yielding nuclear binding energies in agreement within $3 \mathrm{MeV}$.

(2) The rms of the neutron and charge distributions agree in a quite satisfactory way with experimental data. From the set of nuclei studied in this work, systematics of the neutron, proton, charge, and mass rms radii are obtained for spherical nuclei in the form of parametrizations depending on the mass number $A$ and asymmetry $I=(N-Z) / A$ [see Eqs. (17)(20)]. These parametrizations are of the same form as those first proposed in Ref. [8]. The values of the parameters found in the present work are consistent with those derived from experimental data [8] and from the microscopic calculations performed in the framework of the relativistic mean-field approach [6].

(3) Systematics of the ratio $r_{p} / r_{n}$ of the proton $\mathrm{rms}$ to neutron rms radii are of special interest because they are, to a very good approximation, independent of the nuclear deformation and, in addition, they can be used to determine the value of the neutron radius of any nucleus, either spherical or deformed, when its charge radius is known [see Eq. (13)]. The parametrization of $r_{p} / r_{n}$ obtained from the set of nuclei considered in the present study [Eq. (21)] appears in excellent agreement with previous ones based either on experimental data [8] or on the relativistic mean-field approach [6]. This parametrization can therefore be considered as robust enough to be used for predictions concerning nuclei for which experimental data are not yet available, such as nuclei close to the neutron drip line or superheavy elements.

\section{ACKNOWLEDGMENTS}

We wish to express our thanks to Krzysztof Pomorski for the helpful discussions. The careful reading of the manuscript done by Jony Bartel is also acknowledged. B.N.P. is very grateful for the hospitality extended to her by the Service de Physique Nucléaire of the Center d'Etudes de Bruyères-le-Châtel. The work was partially sponsored by the Polish Committee of Scientific Research KBN Grant No. 2P 03B 115 19, POLONIUM Grant No. 01704 UG (2001), and IN2P3 Convention 99-95.
[1] J. Dechargé and D. Gogny, Phys. Rev. C 21, 1568 (1980).

[2] J. F. Berger, M. Girod, and D. Gogny, Comput. Phys. Commun. 63, 365 (1991); Nucl. Phys. A428, 23c (1984).

[3] M. Kleban and B. Nerlo-Pomorska, Annales UMCS AAA LV/ LVI, 1, 2000/2001.

[4] M. Kleban, B. Nerlo-Pomorska, K. Pomorski, J. F. Berger, and J. Dechargé, Acta Phys. Pol. B 32, 1119 (2001).

[5] W. D. Myers and W. J. Świa̧tecki, Nucl. Phys. 81, 1 (1966);
Ark. Fys. 36, 343 (1967).

[6] K. Pomorski, P. Ring, G. A. Lalazissis, A. Baran, Z. Łojewski, B. Nerlo-Pomorska, and M. Warda, Nucl. Phys. A624, 349 (1997).

[7] V. M. Strutinsky, Nucl. Phys. A95, 420 (1967).

[8] B. Nerlo-Pomorska and K. Pomorski, Z. Phys. A 348, 169 (1994).

[9] M. Warda, B. Nerlo-Pomorska, and K. Pomorski, Nucl. Phys. A635, 484 (1998). 
[10] P. Möller, J. P. Nix, and K. Z. Kratz, At. Data Nucl. Data Tables 66, 131 (1997).

[11] S. G. Nilsson, C. F. Tsang, A. Sobiczewski, Z. Szymański, S. Wycech, C. Gustafson, I. L. Lamm, P. Möller, and B. Nilsson, Nucl. Phys. A131, 1 (1969).

[12] K. Pomorski and J. Dudek (private communication).
[13] G. Fricke, C. Bernardt, K. Heiling, L. A. Schaller, L. S. Schellenberg, E. B. Shera, and C. W. De Jager, At. Data Nucl. Data Tables 60, 177 (1995).

[14] C. J. Batty, E. Friedman, H. J. Gils, and H. Rebel, Adv. Nucl. Phys. 19, 1 (1989). 